\title{
A generic linear non-causal optimal control framework integrated with wave excitation force prediction for multi-mode wave energy converters with application to M4
}

\author{
Zhijing Liao ${ }^{\mathrm{a}}$, Peter Stansby ${ }^{\mathrm{b}}$, Guang Lia, ${ }^{\mathrm{a}, *}$ \\ ${ }^{a}$ School of Engineering and Material Science, Queen Mary University of London, London, E1 4NS, UK. \\ ${ }^{b}$ School of Mechanical, Aerospace and Civil Engineering, University of Manchester, Manchester, M13 9PL, UK.
}

\begin{abstract}
The multi-float multi-mode wave energy converter (M-WEC) M4 has essentially linear hydrodynamics characteristics in operational and even extreme waves. This is in contrast to point-absorber and most raft-type devices where nonlinear effects and associated losses are significant. The control problem now involves a large number of degrees of freedom. Energy maximizing control of wave energy converters (WECs) is a non-causal control problem. This paper aims to propose a complete self-contained noncausal optimal control framework by combining a linear non-causal optimal control (LNOC) algorithm with an autoregressive (AR) model as the wave excitation force predictor and a Kalman Filter with random walk wave model (KFRW) as the wave excitation force estimator. The efficacy of the proposed framework together with its enabling components is demonstrated numerically using irregular waves. The proposed framework has low computational load, which enables its real-time implementation on standard computational hardware. Furthermore, the wave force prediction does not require deployment and maintenance of expensive hardware, which helps to reduce the unit cost of the generated electricity.
\end{abstract}

Keywords: Wave excitation force estimation, Kalman filter, Autoregressive model, Optimal control, Non-causal control.

\section{Introduction}

Ocean waves contain considerable renewable energy which can be potentially harvested. Wave energy converters are devices that convert wave energy into electricity but the unit cost of the generated electricity is currently not competitive compared to other types of renewable energy such as wind or solar energy.

Most wave energy converter (WEC) developments have been of point absorber form (e.g. AWS [1], Corpower [2], Seabased [3], Ocean Energy [4]). The idealised power capture due to body motion in regular waves is equal to propagating wave power per metre crest width times wavelength divided by $2 \pi$ for heave motion [1, 2, 3], two times this when operating in surge or pitch and three times this when operating in heave and surge and/or pitch. The theory has been reviewed in [5]. For raft-type devices with two hinged beams the limit is slightly less, shown theoretically in [6]. This determines upper bounds on power capture. There are hybrid raft-type devices with heave, pitch and surge forcing such as Seapower [7], Mocean [8] and the 3-float M4 [9, 10] discussed below.

Linear diffraction-radiation modelling has been widely applied to point absorbers [11] and can significantly overestimate power capture as body motion can be large causing nonlinear effects in reality which reduce motion and power capture [12].

\footnotetext{
${ }^{*}$ Corresponding author

Email addresses: z.liao@qmul.ac.uk (Zhijing Liao), p.k.stansby@manchester.ac.uk (Peter Stansby), g. li@qmul .ac .uk (Guang Li)
}

The modelling may be improved by including nonlinear Froude Krylov and drag forcing [13], [14]. Control has been widely applied to increase power capture showing marked improvements within linear modelling, for a heaving device up to four times magnification in irregular waves in [15]; also for the four degrees of freedom device SEAREV, up to three times in regular waves and two times in irregular waves, compared with no control [16]. Since control generally increases response to improve power capture, the importance of nonlinear effects increases, as discussed for example in [17]. However including fully nonlinear effects can be excessively computationally demanding [18].

To increase power capture the multi-float, multi-mode MWEC system M4 has been developed [9]. There are essentially bow, mid and stern floats, with adjacent floats connected by beams with hinges above the mid floats. There is one bow float and, in its simplest form, one mid and one stern float, of increasing size from bow to stern so that wave drift forces cause alignment with the wave direction. There is one power take off (PTO). Systems with more mid and stern floats, and more hinges, allow more PTOs and greater power capture. Power from forcing modes associated with each float combine constructively [10]. With 8 floats and 4 PTOs capacities similar to offshore wind turbines may be achieved. There is negligible drag due to the rounded or hemi-spherical bases and the system has been shown to be essentially linear [10]. This has been demonstrated for operational conditions by frequency domain modelling for the 3-float device with linear damping [19] and time domain modelling [10]. For the 6-float system with two PTOs, again with linear dampers, linear modelling was seen to slightly underestimate wave basin power measurements [20]. 
The system is thus well designed in not causing the nonlinear effects associated with losses. Wave basin testing for M4 has not been undertaken with control but testing has been undertaken without PTO to determine worst case (large) response in extreme wave conditions. Direct analysis of experimental results [21] and time domain modelling [20] have remarkably shown that response is still predominantly linear. Note this does not extend to extreme mooring forces which can show substantial snatch loads. In extreme motion the addition of a small realistic drag coefficient [22] improves prediction. The M4 WEC, unlike point absorbers and most raft-type devices, is thus an effectively linear system. Optimal control has been applied to the 3-float (single PTO) system assuming known wave conditions and power capture has been increased by $40-100 \%$ over purely linear dampers for a range of sea states [23]. This increase is much less than that claimed for point absorbers but the starting point of the hydrodynamic design is better. Nevertheless the increase is important for commercial viability. With nonlinear hydrodynamic effects insignificant, the major control challenge comes from the multiple degrees of freedom for motions resulting in a model with increased orders (around hundreds or even thousands) compared to that of point absorbers (around 10).

The linear non-causal optimal control (LNOC) method was proposed in [24] aiming to address the energy maximizing problem of WECs by assuming the wave prediction information is available. The control policy contains a feedback part and a feed-forward part to cope with wave prediction. This control method is further extended [23] for the control of the M-WEC, M4, whose dynamics have to be described by a model with a very large dimension. However, in order to implement the LNOC in real-time applications, a practical forward wave excitation force predictor is needed. According to some existing developments [25, 26], the autoregressive (AR) model is a potential candidate. The ocean waves can be treated as a stationary Gaussian process within a certain time period [27]. Under this assumption, the wave excitation force can be described using an AR model. By processing the historical wave excitation force data at every sampling time, the future wave excitation forces can be predicted with reasonable accuracy for a short prediction length.

However, it is generally difficult to measure the wave excitation force not only because load cells are needed but also all hydrodynamic forces are coupled. The wave excitation force needs to be estimated based on other information. A Kalman Filter with a random walk wave model (KFRW) method was proposed in [28] to tackle this issue. By assuming the wave excitation force follows a random walk model, an augmented state vector can be introduced to include both the previous state vector and the wave excitation force term. Then a classical linear Kalman Filter can be used to observe (estimate) the state information and the wave excitation force simultaneously based on the measured WEC motions. With the estimated wave excitation force sequence, the AR predictor can be trained online. In this paper, several critical factors of the AR predictor that may affect the control performance are investigated, e.g. the model order, the training data length and the prediction model update period.
This paper aims to propose a complete control framework as shown in Fig 1 by systematically combining the LNOC, KFRW, and the AR wave excitation force predictor. The combination of WEC control, wave estimation and prediction has also been investigated previously in [15, 29, 30, 31]. In [15], wave prediction requires wave-by-wave measurement by external measurement hardware while the aim of the present study is to investigate the possibility of using measurement of WEC body motion only. In [29], a controller is proposed to cope with significant model uncertainties while the underlying controller is of reactive type (with active tuning of the PTO damping and stiffness coefficient); in terms of utilizing wave prediction, only the dominant frequency of irregular wave data is predicted for control purposes. In [30] a model predictive control method is proposed to deal with operational constraints in the WEC control problem; the AR model is also used for short-term wave prediction and the measurement of historical wave data is assumed to be known. In [31], a hierarchical control framework is proposed to deal with nonlinear WEC dynamics. The framework includes controller, wave estimator and predictor. To cope with nonlinearities the framework has a rather high computational demand and when operational constraints are active the performance degradation is significant. Note that in all cases the point absorber is the only WEC model considered.

It is not straightforward to make comparisons between the different frameworks proposed and the present one, because MWEC is considered in this case instead of a point absorber with single mode motion. The single mode point absorber has rather simple dynamics and low order control model. The model order of M-WEC such as M4 increases significantly due to multiple degrees of freedom and hydrodynamic coupling between different floats, which will have negative impact on online implementation of all three components (controller, wave estimator and predictor). Online optimization becomes more computational demanding. Estimation or prediction failure for any degree of freedom causes failure of the whole framework. These are new challenges compared to the point absorber case. The present control framework, which is designed for M-WECs like M4, has a low computational load due to the linear characteristic of the LNOC. This enables a WEC to be handled with multiple degrees of freedom and thus higher capture width potential. The combination of KFRW and AR predictor is thoroughly investigated from different perspectives. The simulation results suggest that this choice of combination is self-contained and reliable.

The performance of the completed framework is demonstrated by numerical simulations on the M4 WEC model in irregular waves.

The rest of the paper is presented as follows: Section I introduces the control-oriented model of the M4 WEC and the linear non-causal control scheme. Section II presents the KFRW estimator formulation. Section III focuses on details of how the AR model is trained and associated with the non-causal controller. Section IV demonstrates the control performance of the comprehensive control scheme with numerical simulations. Conclusions are drawn in Section V. 


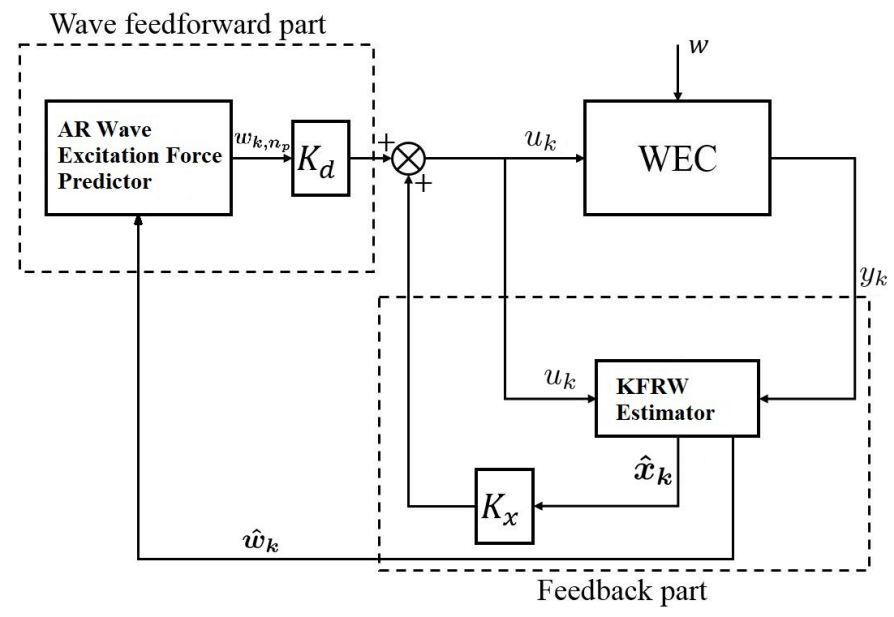

Figure 1: Complete linear non-causal optimal controller framework.

\section{The M4 WEC model and linear non-causal control framework}

\subsection{The M4 WEC}

The M4 wave energy converter was first introduced in [9] as a floating line absorber, and the hydrodynamic design has subsequently been improved [10]. Wave basin experiments were run at different scales to test energy conversion performance and the Froude scaling was confirmed [32]. A linear diffraction model has been built [10] whose fidelity was validated by tank experiment results with a well-tuned linear damper without control. This provides a good basis for validation of the control-oriented model to be derived. Fig 2 shows the simplest 3-float (1-1-1) format of the M4 device at laboratory scale (approximately 1: 40). The floats are named bow float, mid float and stern float from left to right. 1-1-1 indicates their number. Increasing the number of mid and stern floats will increase the number of power take offs (PTOs) but they are governed by the same operating principles. In this paper only uni-directional waves are considered and the waves are assumed to propagate from left to right. The 1-1-1 M4 reacts to wave motion with adjacent floats predominantly in anti-phase to generate relative pitch rotation with respect to the hinge at which a PTO is placed to absorb the kinetic energy. The controller is to be designed to control this PTO actively to maximize the energy output.

\subsection{Control-oriented modelling of the M4 WEC dynamic}

The modelling procedure for a pitch type WEC is similar to the modelling of a point absorber, except that the dimension of WEC motion increases.

Firstly, consider only $x-o-z$ planar motion of the device and choose a generalized coordinate as $q:=\left[x_{o} z_{o} \theta_{1} \theta_{2}\right]^{\top}$. Here $x_{o}$, $z_{o}$ are surge and heave of the hinge $O . \theta_{1}$ and $\theta_{2}$ are the pitch angles of the left and right half of the device, respectively. Then the motion equation can be obtained in the form of

$$
M \ddot{q}(t)=f_{e, q}(t)+f_{p t o, q}(t)-f_{r d, q}(t)-f_{r s, q}(t)
$$

where $f_{r d, q}(t), f_{r s, q}(t), f_{e, q}(t)$ and $f_{p t o, q}(t)$ are the force due to radiation damping, hydrostatic restoring force, wave excitation force based on linear theory and the PTO damping force, respectively.

$M$ is the $4 \times 4$ mass and inertia matrix. Hydrodynamic coefficients are calculated using hydrodynamic software WAMIT [33].

The radiation force can be calculated by the Cummins method [34],

$$
f_{r d, q}(t)=m_{\infty} \ddot{q}(t)+\int_{t-t_{s}}^{t} F_{r d}(t-\tau) \dot{q}(\tau) d \tau
$$

$m_{\infty}$ is the $4 \times 4$ added mass matrix for infinite frequency the diagonal of which can be added to $M . F_{r d}$ is the $4 \times 4$ impulse response function (IRF) matrix and $t_{s}$ is the length of time for each IRF. For a state-space realization, each convolution term can be represented by a transfer function or a state-space model [35]. The order of these subsystems have to be reduced to avoid a huge computational load. The force due to radiation damping can then be expressed as,

$$
\begin{array}{r}
\dot{z}_{s}=A_{s} z_{s}+B_{s} \dot{q}(t) \\
\hat{f}_{r d, q}(t)=C_{s} z_{s}+D_{s} \dot{q}(t)
\end{array}
$$

where $\hat{f}_{r d, q}(t)$ denotes the radiation force calculated by statespace representation.

The hydrostatic restoring force is

$$
f_{r s, q}(t)=K q(t)
$$

where $K$ is the $4 \times 4$ hydrostatic restoring force matrix [10].

The wave excitation force is not modelled since it will be estimated. The PTO force is considered a manipulatable control input. To sum up, the motion equation for M4 can be written as,

$$
\begin{aligned}
\left(M+m_{\infty}\right) \ddot{q}(t)+\hat{f}_{r d, q}(t)+K q(t) & =f_{e, q}(t)+f_{p t o, q}(t) \\
\dot{z}_{s} & =A_{s} z_{s}+B_{s} \dot{q}(t) \\
\hat{f}_{r d, q}(t) & =C_{s} z_{s}+D_{s} \dot{q}(t)
\end{aligned}
$$

By defining a state vector $x:=\left[q, \dot{q}, z_{s}\right]^{T}$, the state-space representation of the M4 control-oriented model can be written as

$$
\begin{aligned}
& \dot{x}=A x+B_{w} f_{e, q}(t)+B_{u} f_{p t o, q}(t) \\
& z=C x
\end{aligned}
$$

where the system matrices are

$$
\begin{aligned}
& A=\left[\begin{array}{ccc}
0_{4 \times 4} & I_{4 \times 4} & 0_{4 \times n} \\
-\left(M+m_{\infty}\right)^{-1} K & -\left(M+m_{\infty}\right)^{-1} D_{s} & -\left(M+m_{\infty}\right)^{-1} C_{s} \\
0_{n \times 4} & B_{s} & A_{s}
\end{array}\right] \\
& B_{w}=\left[\begin{array}{c}
0_{4 \times 4} \\
\left(M+m_{\infty}\right)^{-1} \\
0_{n \times 4}
\end{array}\right] \\
& B_{u}=\left[\begin{array}{c}
0_{4 \times 1} \\
\left(M+m_{\infty}\right)^{-1}[0,0,-1,1]^{\top} \\
0_{n \times 1}
\end{array}\right]
\end{aligned}
$$

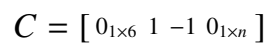




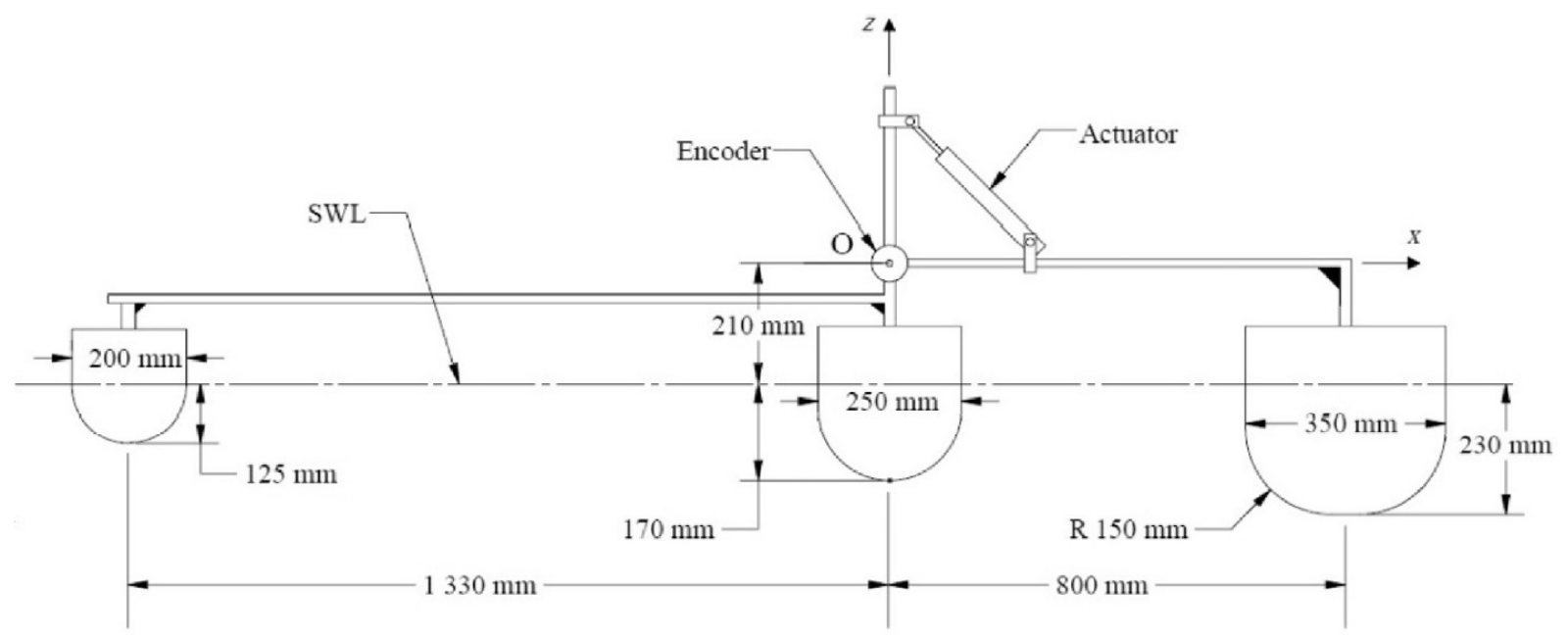

Figure 2: Diagram of laboratory scale three-float M4 1-1-1 from [10.

$n$ is the order of the radiation subsystem. The input matrices $B_{w}$ and $B_{u}$ have different number of columns because excitation force is of dimension 4 and control input is uni-dimensional. Note that for radiation in each degree of freedom, a state-space model of order 20 is relatively accurate, which leads to an $n$ equal to 320 after assembling 16 state-space. The order of the final state-space representation is 328 is this case, which is much higher than that for point-absorbers.

\subsection{Linear non-causal controller}

With the control-oriented model presented in the last section, we can formulate the optimal control problem as follows:

$$
\min _{u_{0}, \ldots, u_{N}} \Sigma_{k=0}^{N}\left\{z_{k} u_{k}+\frac{1}{2} x_{k}^{T} Q x_{k}+\frac{1}{2} R u_{k}^{2}\right\}
$$

subject to the discrete-time state-space model

$$
\begin{aligned}
x_{k+1} & =A x_{k}+B_{w} w_{k}+B_{u} u_{k} \\
z_{k} & =C x_{k}
\end{aligned}
$$

which is discretized from the continuous time model (6). Here $w_{k}$ is the wave excitation force at time $k$ and $u_{k}$ is the control input at time $k$.

$N$ is the number of time steps in the optimization process. In this case $N \rightarrow \infty$. The weighting matrices $Q$ and $R$ are positive definite. They are tuning parameters to balance the tradeoff between the WEC performance, stability of the control system and satisfaction of state constraints and input constraints.

The control law [24] derived from resolving the non-causal optimal control problem presented in (11) has the form of

$$
u_{k}=K_{x} x_{k}+K_{d} w_{k, n_{p}}
$$

which consists of a feedback term for the system states $x_{k}$ and a feed-forward term to incorporate the prediction of the incoming wave excitation force $w_{k, n_{p}}:=\left[w_{k}, w_{k+1}, \ldots, w_{k+n_{p}-1}\right]^{\top}$. Here $n_{p}$ is the length of wave prediction horizon. A non-causal controller means that the future information contributes to the controller's decision making. $K_{x}$ and $K_{d}$ are constant coefficient matrices that can be pre-calculated off-line. According to [24], the formulae for calculating them are

$$
\begin{aligned}
& K_{x}=-\left(R+B_{u}^{T} V B_{u}\right)^{-1}\left(C+B_{u}^{T} V A\right) \\
& K_{d}=-\left(R+B_{u}^{T} V B_{u}\right)^{-1} B_{u}^{T} \Psi
\end{aligned}
$$

and

$$
\begin{gathered}
V=Q+A^{T} V A-\left(C+B_{u}^{T} V A\right)^{T} \\
\left(R+B_{u}^{T} V B_{u}\right)^{-1}\left(C+B_{u}^{T} V A\right)
\end{gathered}
$$

where $V$ is the algebraic Ricatti equation solution. The only unknown is $\Psi$ which is needed to calculate the feedforward gain $K_{d}$. Let $\Phi:=\left(A+B_{u} K_{x}\right)^{T}$, then $\Psi:=$ $\left[V B_{w}, \Phi V B_{w}, \ldots, \Phi^{n_{p}-1} V B_{w}\right]$. Results in [24] show that with an infinite control horizon $N \rightarrow \infty$ and a finite wave prediction horizon $n_{p}$, the control law yields a unique solution.

\section{The Kalman estimator with autoregressive wave model}

In the discrete-time state-space model (12), apart from the wave excitation force $w_{k}$ to be estimated, the system state vector $x_{k}$ is also to be estimated since it contains unmeasurable states corresponding to the radiation subsystem. The system output vector $z_{k}$ consists of the displacement and velocity of the WEC which can be measured by motion sensors. The task of the wave excitation force estimator is to estimate $x_{k}$ and $w_{k}$ based on the information of $z_{k}$ and $u_{k}$.

In the KFRW method, an augmented state vector $\eta_{k}$ is introduced to include both $x_{k}$ and $w_{k}$. To this end, a mathematical model is necessary to perform the time transition of $w_{k}$. A random walk model is adopted in [28] for this purpose,

$$
w_{k+1}=w_{k}+\epsilon_{w, k}
$$


where $\epsilon_{w, k}$ is the random step at time $k$ that $w_{k}$ takes to reach $w_{k+1}$. This is essentially an AR model of order 1 with parameter $\phi_{1}=1$ if $\epsilon_{w, k}$ is considered a zero-mean white noise process that is uncorrelated to any $w_{i}$ with $i<k$. With some minor modifications the system equations and the random walk model can be summarized as,

$$
\begin{aligned}
x_{k+1} & =A x_{k}+B_{w} w_{k}+B_{u} u_{k}+\epsilon_{x, k} \\
w_{k+1} & =w_{k}+\epsilon_{w, k} \\
y_{k} & =z_{k}=C_{k a l} x_{k}+\mu_{k}
\end{aligned}
$$

where $\epsilon_{k}$ represents modelling errors, $y_{k}$ represents the measurement of system output (WEC motions) and $\mu_{k}$ represents the measurement errors. Note that the output matrix $C$ is now $C_{k a l}=\left[I_{8 \times 8}, 0_{8 \times n}\right]^{\top}$ since the displacement and velocity of all dimensions are measurable. Defining the augmented state vector as $\eta_{k}:=\left[x_{k}, w_{k}\right]^{\top}$, the state-space representation of the augmented state can be written as,

$$
\begin{aligned}
\eta_{k+1} & =A_{a} \eta_{k}+B_{a} u_{k}+\epsilon_{k} \\
y_{k} & =z_{k}=C_{a} \eta_{k}+\mu_{k}
\end{aligned}
$$

where $\epsilon_{k}=\left[\epsilon_{x, k}, \epsilon_{w, k}\right]^{\top}$ is the lumped modelling error term. The state transition matrix, input and output matrices are

$$
\begin{aligned}
A_{a} & =\left[\begin{array}{ll}
A & B \\
0 & 1
\end{array}\right] \\
B_{a} & =\left[\begin{array}{c}
B_{u} \\
0
\end{array}\right] \\
C_{a} & =\left[\begin{array}{ll}
C_{k a l} & 0_{8 \times 4}
\end{array}\right]
\end{aligned}
$$

Now the wave excitation force estimation problem becomes a state estimation problem, which can be tackled by a Kalman Filter. By the assumption of a standard Kalman Filter, the model error $\epsilon_{k}$ and the measurement error $\mu_{k}$ should be uncorrelated zero-mean white Gaussian noise process with covariance matrices $Q_{k a l}, R_{k a l}$, respectively, which are tuning parameters to ensure the estimation accuracy.

The KFRW algorithm is summarized briefly for completeness. It takes the control input $u_{k}$ and output $y_{k}$ from measurement as the estimator's inputs, and an estimated state information $\hat{\eta}_{k}$ as the output. It runs at every time step for the estimation.

We denote the former input of the non-causal controller with observer $\hat{u}_{k-1}$, and the estimated state $\hat{\eta}_{k-1}$. Firstly, calculate a priori estimation with the former state information

$$
\hat{\eta}_{k}^{-}=A_{a} \hat{\eta}_{k-1}+B_{a} \hat{u}_{k-1}
$$

and then the error covariance $P_{k}^{-}$of this priori estimation is calculated with the predefined model error covariance $Q_{k a l}$

$$
P_{k}^{-}=A_{a} P_{k-1} A_{a}^{\top}+Q_{k a l}
$$

The Kalman gain is

$$
K_{k a l}=P_{k}^{-} C_{a}^{T}\left(C_{a} P_{k}^{-} C_{a}^{T}+R_{k a l}\right)^{-1}
$$

The estimated state information can be calculated as

$$
\hat{\eta}_{k}=\hat{\eta}_{k}^{-}+K_{k a l}\left(y_{k}-C_{a} \eta_{k}^{-}\right)
$$

The last step in a Kalman algorithm loop is to update the error covariance matrix $P_{k}$ for the next time step

$$
P_{k}=\left(I-K_{k a l} C_{a}\right) P_{k}^{-}
$$

The non-causal optimal control policy with the states estimated by the KFRW estimator can be rewritten as

$$
\hat{u}_{k}=K_{x} \hat{x}_{k}+K_{d} w_{k, n_{p}}
$$

where $\hat{x}_{k}$ is taken from the estimated augmented state $\hat{\eta}_{k}$. The estimated wave excitation force can also be taken from $\hat{\eta}_{k}$ noted as $\hat{w}_{k}$.

In simulation, the measurement of system output $y_{k}$ is set to be the state space model output with added white Gaussian noise (WGN) of Signal-noise-ratio (SNR) equal to $30 \mathrm{~dB}$.

\section{The autoregressive wave force predictor}

In (30), the wave excitation prediction term $w_{k, n_{p}}$ represents the prediction of wave excitation force at time $k$ into future of $n_{p}$ steps provided by the wave predictor.

The autoregressive model is a simple and accurate model used to predict ocean wave elevation or wave excitation force in a short prediction length. The fundamental assumption of an AR model is that the value $w_{k}$ depends linearly on its previous values $w_{k-p}, \ldots, w_{k-1}$, through a set of parameters $\Phi:=$ $\left[\phi_{1}, \ldots, \phi_{p}\right]$. This assumption can be written in the form of

$$
\hat{w}_{k}=\sum_{i=1}^{p} \phi_{i} w_{k-i}
$$

where notation $\hat{w}_{k}$ represents the predicted value of $w_{k} . p$ is the order of the AR model. An AR model of order $p$ is denoted as $\operatorname{AR}(p)$.

To resolve the set of parameters $\Phi$, a set of training data with length $N$ is used to train the model. $\Phi$ can be obtained by minimizing the sum of prediction errors over the training horizon $N$,

$$
J=\sum_{k=p+1}^{N}\left(\eta_{k}-\hat{\eta}_{k}\right)^{2}
$$

which leads to a linear least-square (LLS) problem. The total number of data needed for one training process is $N+p$. With these $N+p$ data the $\operatorname{AR}(p)$ model training process can be written as,

$$
\left[\begin{array}{c}
w_{p+1} \\
w_{p+2} \\
\cdots \\
w_{p+N}
\end{array}\right]=\left[\begin{array}{cccc}
w_{1} & w_{2} & \ldots & w_{p} \\
w_{2} & w_{3} & \ldots & w_{p+1} \\
\ldots & \ldots & \ldots & \ldots \\
w_{N-p} & w_{N-p+1} & \ldots & w_{N-1}
\end{array}\right]\left[\begin{array}{c}
\phi_{1} \\
\phi_{2} \\
\ldots \\
\phi_{p}
\end{array}\right]
$$

or in matrix form

$$
Y=X \Phi
$$


Solving the LLS problem gives the $\operatorname{AR}(p)$ coefficient set as

$$
\Phi=\left(X^{\top} X\right)^{-1} X^{\top} Y
$$

The preview wave excitation force term $w_{k, n_{p}}$ can then be formed by using this $\operatorname{AR}(p)$ model recursively with the past $p$ estimated wave excitation forces,

$$
w_{k, n_{p}}=\left[\hat{w}_{k}, \hat{w}_{k+1}, \ldots, \hat{w}_{k+n_{p}-1}\right]^{\top}
$$

Although the AR model is simple to derive, there are several critical points to be addressed for the wave excitation force prediction purpose.

\section{- Order of the model $p$}

The order of an AR model is the key to its performance. There are several methods proposed to select a suitable order for an autoregressive model, e.g. Akaike's information theoretical criterion [36]. Implementing these methods will inevitably increase the overall computational load because one needs to evaluate the fitness of the model repeatedly and change the model order accordingly. For non-causal control applications, the AR model has to be retrained at a relatively high frequency, so it is more efficient to fix the model order for a longer time. By trail and error we found $p=100$ is a good choice for predicting wave excitation force with JONSWAP wave profile with significant wave height $H_{s}=0.04 m$ and different peak periods; the spectral peakedness factor was unity in all cases. Lower order gives worse prediction and high order does not increase the accuracy. Fig 3 and Fig 4 show the performance comparison between the reference data, $\operatorname{AR}(10)$ and AR(100). Blue vertical line separates the training set and the prediction set.

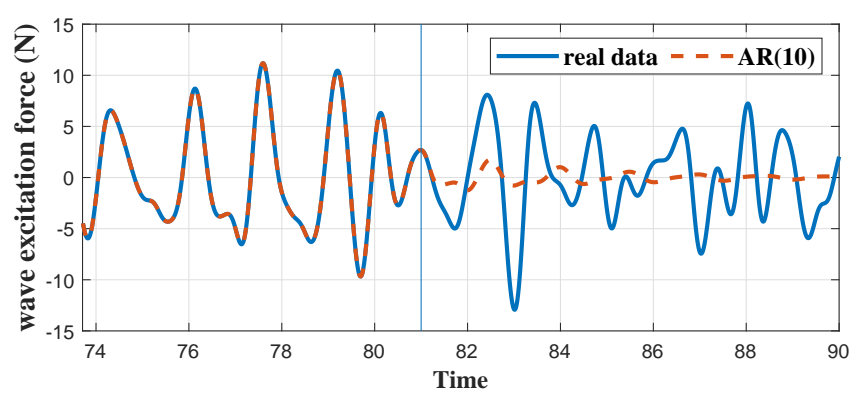

Figure 3: AR model with $p=10, N=800$ to predict wave excitation force with JONSWAP wave peakedness factor $\gamma=1$, significant height $H_{s}=0.04 \mathrm{~m}$ and peak period $T_{p}=1.8 \mathrm{~s}$.

\section{- Training data length $N$}

It is reported in [26] that $N \approx 15 \times p$ gives desired performance and longer training data length does not further improve the prediction accuracy. However, in a discrete time control application with high sample frequency (in this case sampling time $T_{s}=0.009 \mathrm{~s}$ ), the length of training data affects the computation efficiency. We found that by re-sampling the training data with a lower sampling rate,

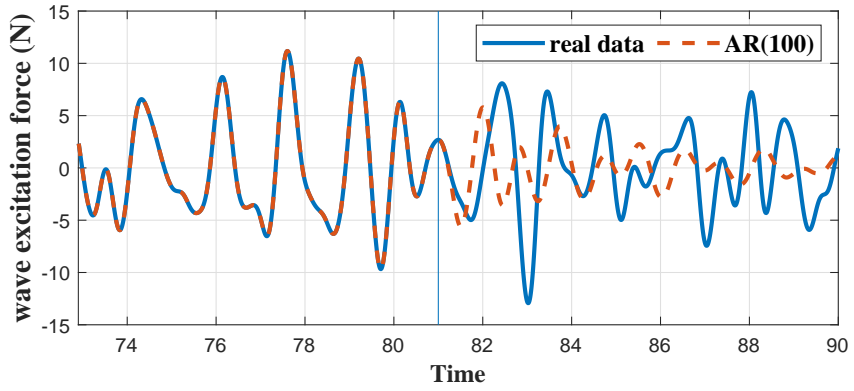

Figure 4: AR model with $p=100, N=800$ to predict wave excitation force with JONSWAP wave peakedness factor $\gamma=1$, significant height $H_{s}=0.04 \mathrm{~m}$ and peak period $T_{p}=1.8 \mathrm{~s}$.

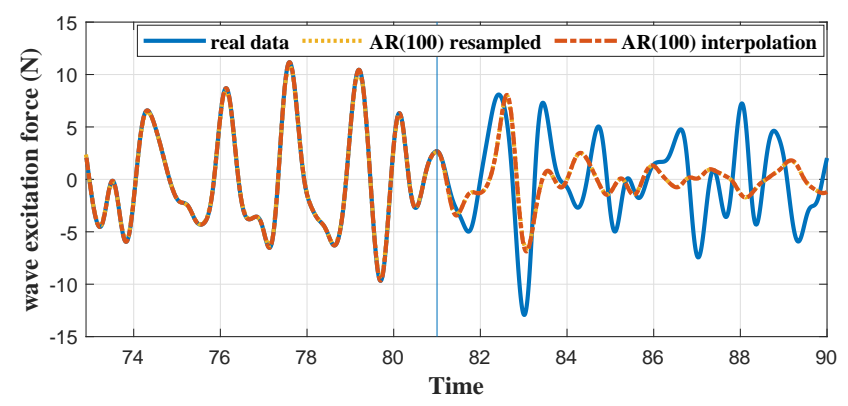

Figure 5: AR model with $p=100, N=8000$ re-sampled to predict wave excitation force with JONSWAP wave peakedness factor $\gamma=1$, significant height $H_{s}=0.04 \mathrm{~m}$ and peak period $T_{p}=1.8 \mathrm{~s}$.

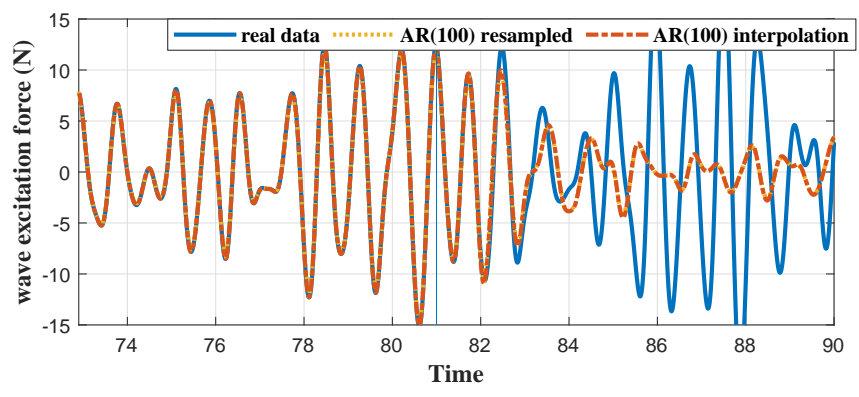

Figure 6: AR model with $p=100, N=8000$ re-sampled to predict wave excitation force with JONSWAP wave peakedness factor $\gamma=1$, significant height $H_{s}=0.04 m$ and peak period $T_{p}=1 \mathrm{~s}$.

which means that fewer training data are used in the training process, the computation load can be reduced while not losing prediction accuracy. Fig 5 shows an AR(100) model with $N=8000$ but re-sampled with $T_{s}=0.09 \mathrm{~s}$ so only 800 sampling data are used. The performance improves to over 2 seconds prediction while computation time remains the same. The lowest re-sampling rate is tested to be the same as the Nyquist frequency of the wave excitation force sequence.

\section{- Retrain period $L$}

Theoretically speaking, the best retraining strategy is to retrain the model every time step since all estimated values are utilized instantly. But technically, it is not computa- 
tionally efficient to do so. So a suitable $L$ should be picked for balancing control performance and computation load. Since the AR(100) can predict accurately up to 2 seconds (with $T_{s}=0.009 s$ it means around 200 samples), we found that to retrain the model with $L=100$ (half of the prediction horizon) time steps is a satisfactory option.

\section{- Prediction horizon $n_{p}$}

Prediction horizon is not a tuning factor of the AR model, but of the controller. However, it will be affected by the AR model performance limitation. For optimal control performance the LNOC requires $2 T_{p}$ prediction horizon which means 3.6 seconds or $n_{p}=400$ time steps in the case of $T_{p}=1.8 \mathrm{~s}$. If the AR model can provide accurate and consistent prediction of $2 T_{p}$ time, the LNOC optimal performance can be maintained. Otherwise, a shorter prediction horizon $n_{p}$ should be chosen.

Fig 5 above shows that at $T_{p}=1.8 \mathrm{~s}$ AR predicts precisely over 2 seconds in the future but less well over 3.6 seconds, so control performance degrades at $T_{p}=1.8 \mathrm{~s}$ is expected. Fig 6 shows a better performance with the same AR model used, but at a different peak period $T_{p}=1 s$. The AR model predicts precisely over 3 seconds. This is more than enough to guarantee optimal control performance. Note that peak periods from 1-1.8 seconds at tank testing scale correspond to peak periods from 6-11 seconds at full scale ocean waves, which are considered the most common sea conditions. Provided that the AR model scales well, the results we obtained here are similar to the published results in [26] reporting that the AR model can predict well for several peak periods into the future.

\section{Numerical simulations}

Numerical simulations are carried out to demonstrate the performance of the LNOC WEC control framework, with comparisons to the LNOC case with ideal wave prediction [23] and the case with a well-tuned passive linear damper. A JONSWAP wave profile of peakedness factor $\gamma=1$ with significant wave height $H_{s}=0.04 m$ is adopted since this was a wave generated also in tank testing of the M4 WEC. Peak periods range from $T_{p}=0.7 \mathrm{~s}$ to $T_{p}=1.8 \mathrm{~s}$ in the simulations. The simulation time is 700 seconds, and the sampling time is set to be $0.009 \mathrm{~s}$.

For the AR wave excitation force predictor, the model order is chosen as $p=100$, data length is $N=800$, the AR predictor is retrained every half peak period, prediction horizon of the LNOC controller is chosen to be $2 \times T_{p}$ seconds. The main focus of the simulation results is to evaluate the degradation of control performance caused by the prediction errors of the AR predictor.

Fig 7 shows the energy output of the WEC. For the passive damper case, energy output is $85.76 J$. The LNOC with ideal prediction case reaches $153.55 \mathrm{~J}$ (79\% improvement) while the LNOC with AR predictor case ends at $130.66 J$ (52\% improvement). Fig 8 shows the corresponding power plot. The better the AR predictor performs, the better the black line follows the

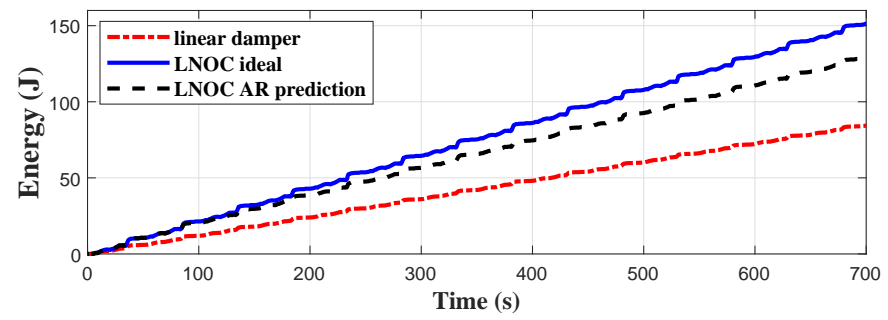

Figure 7: Energy captured, JONSWAP wave profile peakedness factor $\gamma=1$, $H_{s}=0.04 m, T_{p}=1.8 s$.

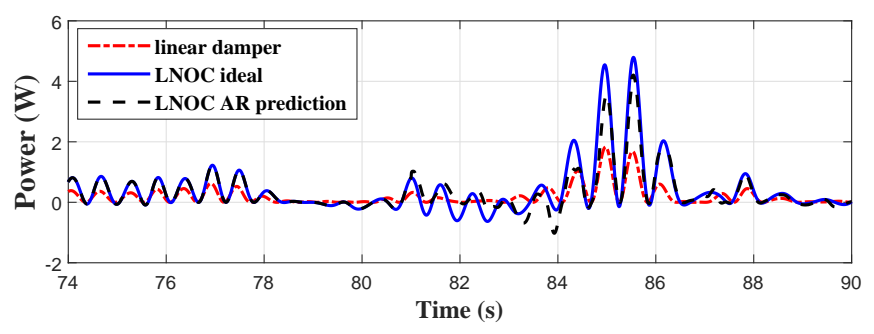

Figure 8: Power, JONSWAP wave profile peakedness factor $\gamma=1, H_{s}=$ $0.04 m, T_{p}=1.8 s$.

ideal blue line in the figure. This can be used as an indicator to evaluate the performance of the AR model. It has been shown in Fig 5 that at around 81 seconds of the simulation, the AR model does not provide accurate prediction up to $2 T_{p}$ time, so in Fig 8 at the same time the black line starts to depart from the ideal blue line. In the meanwhile, the control input in Fig 9 starts to have minor oscillations, while the pitch angle in Fig 10 has smaller amplitude than the ideal case.

The overall control performance for different peak periods can be characterised by the capture width ratio (CWR) plot in Fig 11 . The CWR stands for the ratio between the captured power and the average wave power for one wavelength of crest length. The wavelength corresponding to the energy period, defined here by the wave frequency at the centroid of the spectrum, which collapses CWR data for different spectral peakedness [20]. 12 simulations of different peak periods ranging from $T_{p}=0.7 \mathrm{~s}$ to $T_{p}=1.8 \mathrm{~s}$ are run to evaluate all the CWRs. Significant wave height is fixed at $H_{s}=0.04 \mathrm{~m}$ although this is normalised in CWR. Fig 11 validates that for shorter peak periods, the AR model performance is better, so is the LNOC performance. The overall control performance degradation caused by the inaccuracy of the AR wave excitation force predictor is not significant. This can be also seen from Table 1 by comparing the CWR values of AR prediction and ideal prediction.

Finally, some evaluations on computational load are given to show that the LNOC framework with AR predictor is applicable for real-time implementation. The average computational time for the whole simulation is 0.0035 second which is less than the designed sampling time 0.009 second. For time steps that AR model is retrained, the computational time is roughly 0.1 second. This is larger than the sampling time so the AR model should be retrained at a slower rate separately. Because the retraining happens every half $T_{p}$ time and the AR model can 


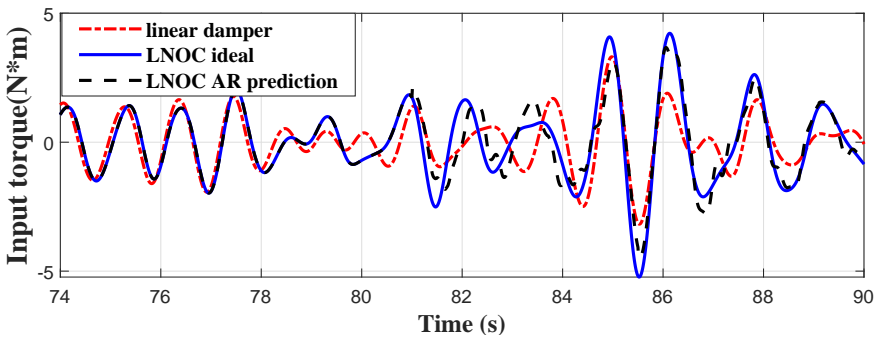

Figure 9: Input torque, JONSWAP wave profile peakedness factor $\gamma=1, H_{s}=$ $0.04 m, T_{p}=1.8 s$.

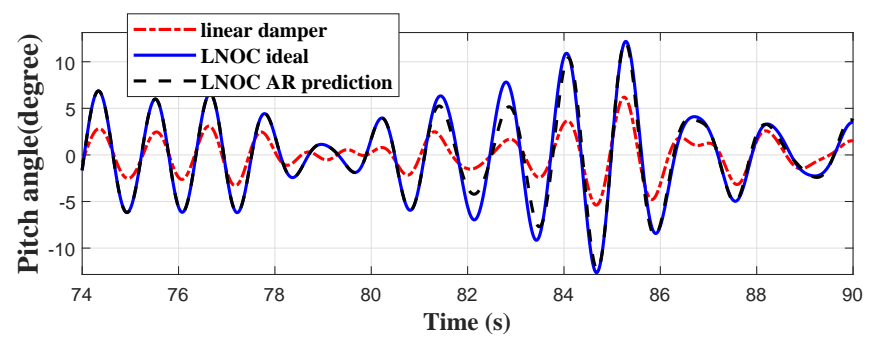

Figure 10: Pitch angle, JONSWAP wave profile peakedness factor $\gamma=1, H_{s}=$ $0.04 m, T_{p}=1.8 s$.

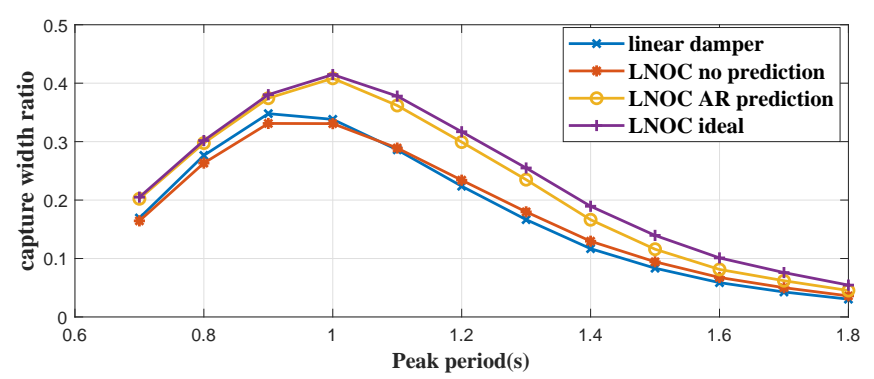

Figure 11: Capture width ratio, JONSWAP peakedness factor $\gamma=1$, wave profile $H_{s}=0.04 m$, wave excitation force prediction horizon is $2 \times T_{p}$.

Table 1: LNOC performance in CWR (percentage)

\begin{tabular}{|l|l|l|l|l|}
\hline $\begin{array}{l}\text { Peak } \\
\text { period }\end{array}$ & $\begin{array}{l}\text { linear } \\
\text { damper }\end{array}$ & $\begin{array}{l}\text { LNOC } \\
\text { no prediction }\end{array}$ & $\begin{array}{l}\text { LNOC } \\
\text { AR prediction }\end{array}$ & $\begin{array}{l}\text { LNOC } \\
\text { ideal prediction }\end{array}$ \\
\hline $0.7 \mathrm{~s}$ & $16.9 \%$ & $16.4 \%$ & $20.2 \%$ & $20.5 \%$ \\
\hline $0.8 \mathrm{~s}$ & $27.6 \%$ & $26.3 \%$ & $30.0 \%$ & $30.2 \%$ \\
\hline $0.9 \mathrm{~s}$ & $34.8 \%$ & $33.1 \%$ & $37.4 \%$ & $38.0 \%$ \\
\hline $1.0 \mathrm{~s}$ & $33.8 \%$ & $33.1 \%$ & $40.8 \%$ & $41.5 \%$ \\
\hline $1.1 \mathrm{~s}$ & $28.6 \%$ & $28.9 \%$ & $36.2 \%$ & $37.8 \%$ \\
\hline $1.2 \mathrm{~s}$ & $22.4 \%$ & $23.4 \%$ & $30.0 \%$ & $31.7 \%$ \\
\hline $1.3 \mathrm{~s}$ & $16.7 \%$ & $18.0 \%$ & $23.5 \%$ & $25.5 \%$ \\
\hline $1.4 \mathrm{~s}$ & $11.7 \%$ & $13.0 \%$ & $16.6 \%$ & $18.9 \%$ \\
\hline $1.5 \mathrm{~s}$ & $8.3 \%$ & $9.4 \%$ & $11.6 \%$ & $14.0 \%$ \\
\hline $1.6 \mathrm{~s}$ & $5.9 \%$ & $6.8 \%$ & $8.1 \%$ & $10.1 \%$ \\
\hline $1.7 \mathrm{~s}$ & $4.3 \%$ & $5.0 \%$ & $6.2 \%$ & $7.6 \%$ \\
\hline $1.8 \mathrm{~s}$ & $3.0 \%$ & $3.6 \%$ & $4.5 \%$ & $5.5 \%$ \\
\hline
\end{tabular}

predict up to $2 \times T_{p}$ time, the computational time of $0.1 \mathrm{~s}$ is acceptable. For time steps that retraining does not happens, the computational time is trivial since the controller is computed off-line. Note that at full scale the time scale is at least 6 times longer than the laboratory scale, so real time control becomes even easier.

\section{Conclusion}

With a M-WEC such as M4, power from modes associated with each float may combine constructively to increase overall power capture. The hydrodynamic system has been shown to be essentially linear but with a large number of degrees of freedom which presents the control challenge. A complete self-contained non-causal optimal control frame work for such WEC control is proposed. With a Kalman Filter estimator and the well-known AR model wave excitation force predictor, the LNOC control performance is investigated. We found that the AR model predictor can provide satisfactory prediction of the incoming wave excitation force for control purpose. Tuning of the AR model order and the training data length are both critically important for the AR predictor. The LNOC control framework is robust against prediction errors and has small computational load. Similar models could be applied to other multibody problems such as control of vessels accessing offshore platforms and of ship-to-ship offloading.

\section{Acknowledgement}

Zhijing Liao is grateful to the financial support from the joint "Queen Mary University of London - China Scholarship Council" scholarship.

This work was also supported in part by a research contract from Wave Energy Scotland's Control Systems programme and in part by the Newton Advanced Fellowship (No. NA160436) from Royal Society.

\section{References}

[1] AWS Ocean Energy. http://www . awsocean.com/ [Online; accessed 23-September-2019].

[2] CorPower Ocean. http://www.corpowerocean.com/ [Online; accessed 23-September-2019].

[3] Seabased. https://www.seabased.com/ [Online; accessed 23September-2019].

[4] OceanEnergy. http://www.oceanenergy.ie/ [Online; accessed 23September-2019].

[5] J. Falnes. Ocean Waves and Oscillating Systems: Linear Interactions Including Wave-Energy Extraction. Cambridge University Press, 2002.

[6] J.N. Newman. Absorption of wave energy by elongated bodies. Applied Ocean Research, 1(4):189 - 196, 1979.

[7] Sea Power. http://www.seapower.ie/ [Online; accessed 23September-2019].

[8] Mocean Energy. https://www . mocean.energy [Online; accessed 23September-2019].

[9] P. Stansby, E. Carpintero Moreno, T. Stallard, and A. Maggi. Three-float broad-band resonant line absorber with surge for wave energy conversion. Renewable Energy, 78:132 - 140, 2015.

[10] P. Stansby, E. Carpintero Moreno, and T. Stallard. Large capacity multifloat configurations for the wave energy converter $\mathrm{m} 4$ using a timedomain linear diffraction model. Applied Ocean Research, 68:53 - 64, 2017.

[11] A. F. de O. Falcão. Wave energy utilization: A review of the technologies. Renewable and Sustainable Energy Reviews, 14(3):899 - 918, 2010.

[12] M. Penalba, G. Giorgi, and J. V. Ringwood. Mathematical modelling of wave energy converters: A review of nonlinear approaches. Renewable and Sustainable Energy Reviews, 78:1188 - 1207, 2017.

[13] M. Penalba and J. V. Ringwood. Linearisation-based nonlinearity measures for wave-to-wire models in wave energy. Ocean Engineering, $171: 496-504,2019$. 
[14] G. Giorgi and J. V. Ringwood. Comparing nonlinear hydrodynamic forces in heaving point absorbers and oscillating wave surge converters. Journal of Ocean Engineering and Marine Energy, 4(1):25-35, Feb 2018.

[15] U. A. Korde. Near-optimal control of a wave energy device in irregular waves with deterministic-model driven incident wave prediction. Applied Ocean Research, 53:31 - 45, 2015.

[16] A. Babarit and A.H. Clément. Optimal latching control of a wave energy device in regular and irregular waves. Applied Ocean Research, 28(2):77 $-91,2006$.

[17] J. Davidson, C. Windt, G. Giorgi, R. Genest, and J. V. Ringwood. Evaluation of Energy Maximising Control Systems for Wave Energy Converters Using OpenFOAM, pages 157-171. Springer International Publishing, Cham, 2019.

[18] M. Penalba, J. Davidson, C. Windt, and J. V. Ringwood. A high-fidelity wave-to-wire simulation platform for wave energy converters: Coupled numerical wave tank and power take-off models. Applied Energy, 226:655 $-669,2018$.

[19] L. Sun, J. Zang, P. Stansby, E. Carpintero Moreno, P.H. Taylor, and R Eatock-Taylor. Linear diffraction analysis of the three-float multi-mode wave energy converter $\mathrm{m} 4$ for power capture and structural analysis in irregular waves with experimental validation. Journal of Ocean Engineering and Marine Energy, 3(1), 2017.

[20] E. Carpintero Moreno and P. Stansby. The 6-float wave energy converter $\mathrm{m} 4$ : ocean basin tests giving capture width, response and energy yield for several sites. Renewable and Sustainable Energy Reviews, 104:307 - 318, 2019.

[21] H. Santo, P. H. Taylor, E. Carpintero Moreno, P. Stansby, R. Eatock Taylor, L. Sun, and J. Zang. Extreme motion and response statistics for survival of the three-float wave energy converter $\mathrm{m} 4$ in intermediate water depth. Journal of Fluid Mechanics, 813:175-204, 2017.

[22] H. Gu, P. Stansby, T. Stallard, and E. Carpintero Moreno. Drag, added mass and radiation damping of oscillating vertical cylindrical bodies in heave and surge in still water. Journal of Fluids and Structures, 82:343-356, 102018.

[23] Z. Liao, N. Gai, P. Stansby, and G. Li. Linear non-causal optimal control of an attenuator type wave energy converter m4. IEEE Transactions on Sustainable Energy, pages 1-1, 2019.

[24] S. Zhan and G. Li. Linear optimal noncausal control of wave energy converters. IEEE Transactions on Control Systems Technology, 27(4):15261536, July 2019.

[25] F. Fusco and J. V. Ringwood. Short-term wave forecasting for real-time control of wave energy converters. IEEE Transactions on Sustainable Energy, 1(2):99-106, July 2010.

[26] Y. Peña-Sanchez, A. Mérigaud, and J. V. Ringwood. Short-term forecasting of sea surface elevation for wave energy applications: The autoregressive model revisited. IEEE Journal of Oceanic Engineering, pages 1-10, 2018.

[27] M. K. Ochi. Ocean waves : the stochastic approach. Cambridge, U.K. ; New York : Cambridge University Press, 1998. Includes bibliographical references and index.

[28] H. . Nguyen and P. Tona. Wave excitation force estimation for wave energy converters of the point-absorber type. IEEE Transactions on Control Systems Technology, 26(6):2173-2181, Nov 2018.

[29] M. P. Schoen, J. Hals, and T. Moan. Wave prediction and robust control of heaving wave energy devices for irregular waves. IEEE Transactions on Energy Conversion, 26(2):627-638, June 2011.

[30] P. Tona, H-N Nguyen, G. Sabiron, and Y. Creff. An Efficiency-Aware Model Predictive Control Strategy for a Heaving Buoy Wave Energy Converter. In 11th European Wave and Tidal Energy Conference - EWTEC 2015, Nantes, France, September 2015.

[31] A. Mérigaud and J. V. Ringwood. Towards realistic non-linear recedinghorizon spectral control of wave energy converters. Control Engineering Practice, 81:145 - 161, 2018.

[32] P. Stansby, E. Carpintero Moreno, and T. Stallard. Capture width of the three-float multi-mode multi-resonance broadband wave energy line absorber $\mathrm{m} 4$ from laboratory studies with irregular waves of different spectral shape and directional spread. Journal of Ocean Engineering and Marine Energy, 1(3):287-298, Aug 2015.

[33] C.H. Lee and J. N. Newman. Wamit - user manual version 7.0. In WAMIT Inc, Chestnut Hill, Massachusetts, 2013.

[34] W.E.Cummins. The impulse response function and ship motions.
[35] E. Kristiansen, A. Hjulstad, and O. Egeland. State-space representation of radiation forces in time-domain vessel models. Ocean Engineering, 32(17):2195 - 2216, 2005.

[36] H. Akaike. A new look at the statistical model identification. IEEE Transactions on Automatic Control, 19(6):716-723, December 1974 